\title{
COMPARATIVE ANALYSIS OF RIEMANN-LIOUVILLE, CAPUTO-FABRIZIO, AND ATANGANA-BALEANU INTEGRALS
}

\author{
Asif Ali Shaikh ${ }^{1}$, Sania Qureshi ${ }^{1,2}$ \\ ${ }^{1}$ Department of Basic Sciences and Related Studies, Mehran University \\ of Engineering and Technology, \\ Jamshoro, 76062, Pakistan \\ ${ }^{2}$ Department of Mathematics, Near East University TRCN, Mersin 10, Turkey \\ asif.shaikh@faculty.muet.edu.pk, sania.qureshi@faculty.muet.edu.pk
}

Received: 2 November 2021; Accepted: 31 December 2021

\begin{abstract}
This study analyzes the most commonly used operators of the Riemann-Liouville, the Caputo-Fabrizio, and the Atangana-Baleanu integral operators. Firstly, a numerical scheme for the Riemann-Liouville fractional integral has been discussed. Then, two numerical techniques have been suggested for the remaining two operators. The experimental order of convergence for the schemes is further supported by the computations of absolute relative error at the final nodal point over the integration interval $[0, T]$. Comparative analysis of the integrals reveals that the Riemann-Liouville fractional integral yields the most minor errors and the most significant experimental order of convergence in the majority of functions, particularly when the fractional-order parameter $\alpha \rightarrow 0$. It is worth noting that the Atangana-Baleanu has proved to be an essential operator for solving many dynamical systems that a single RL operator cannot handle. All of the three integral operators coincide with each other for $\alpha=1$. Mathematica 11.3 for an Intel(R) Core(TM) i3-4500U processor running on $1.70 \mathrm{GHz}$ is used to carry out all the necessary computations.
\end{abstract}

MSC 2010: 65M06, 26A33

Keywords: fractional operator, gamma function, absolute error, convergence

\section{Introduction}

Fractional calculus (differentiation and integration of order $\alpha \in \mathbb{R}^{+}$), earlier considered to be a branch of pure mathematical analysis, has now been found to be extremely useful in various fields of applied sciences. It plays a vital role in modeling diverse physical and natural phenomena including neural networks [1], bio-electrodes [2], bio-materials [3], mathematical biology and bifurcations [4-7], finance and economics [8], electrical and mechanical engineering [9-15], fluid dynamics [16], control systems [17], plant genetics [18-20] and many more. One of the major reasons for the rapidly increasing popularity of this field is its capability of modeling all those dynamic systems which have history (memory) effects and anomalous behavior, which is something common in most of the physical and natural 
systems. On the other hand, integer-order calculus (differentiation and integration of order $n \in \mathbb{N}$ ) does not have such capabilities due to minimal degrees of freedom for the integer-order parameter $n \in \mathbb{N}$.

Among many, the most commonly used approach for computing a fractional-order integral is the Riemann-Liouville fractional integral, which is a straightforward generalization of the Cauchy formula for repeated integration from classical calculus. However, in the last half-decade, various approaches for fractional-order derivatives and integrals have been proposed. The reasons for the birth of these new approaches is considered to be the non-singularity that is present at one end-point of the integration interval $[0, T]$ in the Riemann-Liouville integral. The new approaches try to avoid such singularity. The first such attempt was taken by Caputo and Fabrizio in [21] in which the integral involved is non-singular and depends on the decaying exponential smooth function. In an attempt to modify this approach, Atanagana-Baleanu suggested an approach in [22] and replaced an exponential smooth function by a more generalized function called the Mittag-Leffler function with one-parameter:

$$
E_{\alpha}(z):=\sum_{n=0}^{\infty} \frac{z^{n}}{\Gamma(\alpha n+1)}, \alpha>0, z \in \mathbb{R} .
$$

In the light of the vitality of the fractional operators in different fields as mentioned above and in exhibiting the memory effects of the dynamical systems, we feel motivated to under-seek the impact of integral operators for Riemann-Liouville, Caputo-Fabrizio and the Atangana-Baleanu through comparative analysis and numerical representations. As a consequence, we compute and provide the outlook of the exact solutions through these approaches, and the associated behavior of the absolute relative errors are plainly exhibited. Moreover, the numerical schemes have been suggested for these integrals, and the experimental order of convergence is computed.

The present paper is structured as follows: section 2 and its corresponding subsections provide the discussion of the three types of integrals under consideration and derive the numerical schemes. Section 3 is devoted to describing the obtained results and presenting the analysis carried out in the tabular data. Finally, the paper is concluded in section 4 with a discussion of some significant findings.

\section{Fractional integrals}

In this section, three different types of integrals under Riemann-Liouville (RL), Caputo-Fabrizio (CF), and the Atangana-Baleanu (AB) have been considered. Firstly, the RL fractional integral of a function with fractional-order parameter $\alpha>0$ has been numerically evaluated in an explicit and step-by-step manner. It may be noted that such evaluation is described in [23] but not presented in its simplified form as the one considered in the present study. After this evaluation, the CF integral is numerically given. Lastly, we consider the $\mathrm{AB}$ integral operator and use the RL fractional integral to get a numerical scheme for $\mathrm{AB}$. 


\subsection{Riemann-Liouville Fractional Integral}

Definition 1 [23] A well-defined integral called the Riemann-Liouville fractional integral of order $\alpha>0$ for a locally integrable real function $y: \mathbb{R} \rightarrow \mathbb{R}$ is defined by:

$$
\mathrm{RL}_{0, t}^{\alpha} y(t)=\frac{1}{\Gamma(\alpha)} \int_{0}^{t}(t-\tau)^{\alpha-1} y(\tau) d \tau, t>0,
$$

where $\Gamma(\cdot)$ is the Euler's gamma function.

To begin with, suppose that $y(t) \in C[0, T]$ and $h$ are the step length with $h=\frac{T}{n}$ with $n \in \mathbb{N}$ and denote $t_{k}=k h$. Next, consider the Riemann Liouville fractional integral (2) at $t=t_{n}, n \in \mathbb{N}$ to obtain the following:

$$
\begin{aligned}
& \left.\mathrm{RL}_{0, t}^{\alpha} y(t)\right|_{t=t_{n}}=\frac{1}{\Gamma(\alpha)} \int_{0}^{t_{n}}\left(t_{n}-\tau\right)^{\alpha-1} y(\tau) d \tau, \alpha \in(0,1], \quad t>0, \\
& \left.\operatorname{RL}_{0, t}^{\alpha} y(t)\right|_{t=t_{n}}=\frac{1}{\Gamma(\alpha)}\left[\int_{0}^{t_{1}}\left(t_{n}-\tau\right)^{\alpha-1} y(\tau) d s+\int_{t_{1}}^{t_{2}}\left(t_{n}-\tau\right)^{\alpha-1} y(\tau) d \tau+\ldots+\int_{t_{n-1}}^{t_{n}}\left(t_{n}-\tau\right)^{\alpha-1} y(\tau) d \tau\right], \\
& \left.\operatorname{RL}_{0, t}^{\alpha} y(t)\right|^{=}=\frac{1}{\Gamma(\alpha)} \sum_{t=t_{n}}^{n-1} \int_{t_{k}}^{t_{k+1}}\left(t_{n}-\tau\right)^{\alpha-1} y(\tau) d \tau, t_{0}=0 .
\end{aligned}
$$

Using the classical left rectangular rule for integration, one can replace $y(t)$ by a piece-wise constant interpolant on the integration interval $[0, T]=\left[t_{k}, t_{k+1}\right]$, $k=0,1,2, \ldots, n-1$ to obtain the following:

$$
\left.y(t)\right|_{\left[t_{k}, t_{k+1}\right)} \approx y\left(t_{k}\right) .
$$

Thus, one obtains

$$
\left.\mathrm{RL}_{0, t}^{\alpha} y(t)\right|_{t=t_{n}} \approx \frac{1}{\Gamma(\alpha)} \sum_{k=0}^{n-1} \int_{t_{k}}^{t_{k+1}}\left(t_{n}-\tau\right)^{\alpha-1} y\left(t_{k}\right) d \tau \approx \frac{1}{\Gamma(\alpha)} \sum_{k=0}^{n-1} y\left(t_{k}\right) \int_{t_{k}}^{t_{k+1}}\left(t_{n}-\tau\right)^{\alpha-1} d \tau .
$$

Integration with respect to $\tau$ yields

$$
\begin{aligned}
& \left.\operatorname{RL}_{0, t}^{\alpha} y(t)\right|_{t=t_{n}} \approx \frac{1}{\Gamma(\alpha)} \sum_{k=0}^{n-1} y\left(t_{k}\right)\left[\frac{\left(t_{n}-\tau\right)^{\alpha}}{-\alpha}\right]_{t_{k}}^{t_{k+1}} \approx \frac{1}{\alpha \Gamma(\alpha)} \sum_{k=0}^{n-1} y\left(t_{k}\right)\left[\left(t_{n}-t_{k}\right)^{\alpha}-\left(t_{n}-t_{k+1}\right)^{\alpha}\right], \\
& \left.\operatorname{RL}_{0, t}^{\alpha} y(t)\right|_{t=t_{n}} \approx \frac{h^{\alpha}}{\Gamma(\alpha+1)} \sum_{k=0}^{n-1} y\left(t_{k}\right)\left[(n-k)^{\alpha}-(n-k-1)^{\alpha}\right],
\end{aligned}
$$


where $t_{n}=n h, t_{k}=k h$ and $t_{k+1}=(k+1) h$. For $n=1,2$, and 3, one obtains the following

$$
\begin{gathered}
\left.\operatorname{RL}_{0, t}^{\alpha} y(t)\right|_{t=t_{1}} \approx \frac{h^{\alpha}}{\Gamma(\alpha+1)} y\left(t_{0}\right) . \\
\left.\operatorname{RL}_{0, t}^{\alpha} y(t)\right|_{t=t_{2}} \approx \frac{h^{\alpha}}{\Gamma(\alpha+1)}\left[y\left(t_{0}\right)\left(2^{\alpha}-1\right)+y\left(t_{1}\right)\right] . \\
\left.\operatorname{RL}_{0, t}^{\alpha} y(t)\right|_{t=t_{3}} \approx \frac{h^{\alpha}}{\Gamma(\alpha+1)}\left[y\left(t_{0}\right)\left(3^{\alpha}-2^{\alpha}\right)+y\left(t_{1}\right)\left(2^{\alpha}-1\right)+y\left(t_{2}\right)\right] .
\end{gathered}
$$

Continuing in the same way, one obtains

$$
\left.\mathrm{RL}_{0, t}^{\alpha} y(t)\right|_{t=t_{n}} \approx \frac{h^{\alpha}}{\Gamma(\alpha+1)}\left[y\left(t_{0}\right)\left(n^{\alpha}-(n-1)^{\alpha}\right)+\ldots+y\left(t_{n-3}\right)\left(3^{\alpha}-2^{\alpha}\right)+y\left(t_{n-2}\right)\left(2^{\alpha}-1\right)+y\left(t_{n-1}\right)\right] .
$$

Alternatively, one obtains the following scheme

$$
\left.{ }^{\mathrm{RL}} J_{0, t}^{\alpha} y(t)\right|_{t=t_{n}} \approx \frac{h^{\alpha}}{\Gamma(\alpha+1)} \sum_{k=0}^{n-1}\left[(k+1)^{\alpha}-k^{\alpha}\right] y\left(t_{n-k-1}\right) .
$$

This scheme is presented in a simplified form to numerically evaluate the RL integral of $y(t)$. It may be noted that when $\alpha=1$ in RL integral (2), one retrieves the ordinary integral from the classical calculus. For the error bounds of the above numerical scheme in which a substantial amount of quadrature theory is involved, we state the following theorem whose proof can be found in [24].

Theorem 1 Suppose that $y \in C^{1}[0, T]$. Then

$$
\left|\int_{0}^{t_{n}}\left(t_{n}-t\right)^{\alpha-1} y(t) d t-\sum_{k=0}^{n-1}\left((k+1)^{\alpha}-k^{\alpha}\right) y\left(t_{n-k-1}\right)\right| \leq \frac{h}{\alpha} \| y^{\prime}|| t_{n}^{\alpha}
$$

Further, suppose that $y(t)=t^{q}$ for some $0<q<1$. Then

$$
\left|\int_{0}^{t_{n}}\left(t_{n}-t\right)^{\alpha-1} y(t) d t-\sum_{k=0}^{n-1}\left((k+1)^{\alpha}-k^{\alpha}\right) y\left(t_{n-k-1}\right)\right| \leq h R_{\alpha, q} t_{n}^{\alpha+q-1},
$$

where $R_{\alpha, q}$ is some constant that depends only on $\alpha$ and $q$.

Thus, looking at the well-established error analysis for the Riemann-Liouville fractional integral, it is safe to use this integral to study the recently proposed integrals as discussed in the present study. 


\subsection{Caputo-Fabrizio integral}

Definition 2 [21] The CF integral of order $\alpha>0$ for a function $y \in \mathbb{H}^{1}(0, T)$ with $T>0$ is defined by

$$
\mathrm{CF}_{0, t}^{\alpha} y(t)=\frac{2(1-\alpha)}{(2-\alpha) M(\alpha)} y(t)+\frac{2 \alpha}{(2-\alpha) M(\alpha)} \int_{0}^{t} y(\tau) d \tau, t>0,
$$

where $M(\alpha)$ is a normalizing smooth function.

The above definition can be rewritten as follows:

$$
{ }^{\mathrm{CF}} J_{0, t}^{\alpha} y(t)=\frac{2}{(2-\alpha) M(\alpha)}[(1-\alpha) y(t)+\alpha J(y(t))],
$$

where $J(y(t))$ is the ordinary integral. Furthermore, in order to retrieve the ordinary integral when $\alpha=1$ in the CF definition, we impose the following condition:

$$
M(\alpha)=\frac{2}{2-\alpha} .
$$

In order to get numerical scheme for the CF integral presented in (15), the ordinary integral involved in it is approximated by the first order left rectangular formula using classical numerical analysis. The resultant numerical scheme becomes

$$
\left.\mathrm{CF}_{0, t}^{\alpha} y(t)\right|_{t=t_{n}} \approx \frac{2}{(2-\alpha) M(\alpha)}\left[(1-\alpha) y\left(t_{n-1}\right)+\alpha h \sum_{k=0}^{n-1} y\left(t_{k}\right)\right] .
$$

This scheme is presented in a simplified form to numerically evaluate the CF integral of $y(t)$. It may be noted that when $\alpha=1$ and $M(\alpha)$ from (16) in CF integral (14), one retrieves the ordinary integral from the classical calculus. In addition to this, the upper bound for left rectangular rule from classical quadrature is found to be $\left|e_{n}\right| \leq \frac{T^{2}\left|y^{\prime}(t)\right|}{2 n}$, where $n$ is the number of integration steps along the interval $[0, T]$. A detailed error analysis can also be found in [25].

\subsection{Atanagana-Baleanu integral}

Definition 3 [22] The AB integral of order $\alpha>0$ for a function $y \in \mathbb{H}^{1}(0, T)$ with $T>0$ is defined as

$$
{ }^{\mathrm{AB}} J_{0, t}^{\alpha} y(t)=\frac{1-\alpha}{B(\alpha)} y(t)+\frac{\alpha}{B(\alpha) \Gamma(\alpha)} \int_{0}^{t}(t-\tau)^{\alpha-1} y(\tau) d \tau,
$$

where $B(\alpha)=1-\alpha+\frac{\alpha}{\Gamma(\alpha)}$ is a normalizing constant function depending upon $\alpha$. Further simplifying (18), one obtains

$$
{ }^{\mathrm{AB}} J_{0, t}^{\alpha} y(t)=\frac{1}{B(\alpha)}\left((1-\alpha) y(t)+\alpha^{\mathrm{RL}} J_{0, t}^{\alpha} y(t)\right) .
$$


Upon using the numerical scheme for RL as obtained in (11), one gets

$$
\left.{ }^{\mathrm{AB}} J_{0, t}^{\alpha} y(t)\right|_{t=t_{n}} \approx \frac{1}{B(\alpha)}\left[(1-\alpha) y\left(t_{n-1}\right)+\frac{h^{\alpha}}{\Gamma(\alpha)} \sum_{k=0}^{n-1}\left((k+1)^{\alpha}-k^{\alpha}\right) y\left(t_{n-k-1}\right)\right] .
$$

This scheme is presented in a simplified form to numerically evaluate the AB integral of $y(t)$. It may be noted that when $\alpha=1$ in AB integral (18), one retrieves the ordinary integral from the classical calculus.

\section{Results and discussion}

This section is devoted to employing the schemes presented in (11), (17) and (20) for solving the integrals under Riemann-Liouville, Caputo-Fabrizio, and the Atangana-Baleanu operators, respectively. The nature of the functions varies from polynomial to transcendental, and their exact solutions consist of the gamma function and its types. Tabular data represent the computation of the absolute relative errors at final nodal point $\left(E(t=T)=\left|\frac{y(T)-y_{N}}{y(T)}\right|\right)$ of the associated integration interval. In addition to this, the value for the experimental order of convergence computed as EOC $=\log _{2}\left(\frac{E(h)}{E(h / 2)}\right)$ demonstrates the first-order accuracy of the schemes under consideration where $h$ denotes the step length used for the respective numerical scheme.

Example 1 Consider $y(t)=t^{2}, t \geq 0$ as the first function to be analyzed. The exact solution under each integral operator is shown below:

$$
\begin{gathered}
{ }^{\mathrm{RL}} J_{0, t}^{\alpha} y(t)=\frac{2 t^{\alpha+2}}{\alpha(\alpha+1)(\alpha+2) \Gamma(\alpha)} . \\
{ }^{\mathrm{CF}} J_{0, t}^{\alpha} y(t)=\frac{2\left(1-\alpha+\frac{\alpha}{3} t\right) t^{2}}{(2-\alpha) M(\alpha)} . \\
{ }^{\mathrm{AB}} J_{0, t}^{\alpha} y(t)=\frac{\left(2 t^{\alpha}-\left(\alpha^{2}-1\right)(\alpha+2) \Gamma(\alpha)\right) t^{2}}{(\alpha+1)(\alpha+2) B(\alpha) \Gamma(\alpha)} .
\end{gathered}
$$

For this polynomial function, one can observe from Table 1 that the absolute relative error computed at the final nodal point $(t=1)$ for an RL fractional integral operator is smallest for varying values of the fractional-order parameter $\alpha$. Moreover, by decreasing the step length $h$ by one order of magnitude, we get one order decrease in the tenth power of the errors under every integral operator as shown in Table 1. This suggests the first-order convergence of the numerical schemes, which is further 
confirmed with computation of experimental order of convergence in the Table 2 which is always $\approx 1$ but largest in case of RL fractional integral.

Table 1. Absolute relative errors at final nodal point over $[0,1]$ for the example 1

(a) $\alpha=0.25$

\begin{tabular}{c|c|c|c}
\hline$h$ & $10^{-1}$ & $10^{-2}$ & $10^{-3}$ \\
\hline RL & $1.5522 \mathrm{e}-01$ & $1.4035 \mathrm{e}-02$ & $1.2847 \mathrm{e}-03$ \\
\hline CF & $1.8550 \mathrm{e}-01$ & $1.9405 \mathrm{e}-02$ & $1.9490 \mathrm{e}-03$ \\
\hline AB & $1.8279 \mathrm{e}-01$ & $1.8684 \mathrm{e}-02$ & $1.8509 \mathrm{e}-03$ \\
\hline
\end{tabular}

(c) $\alpha=0.75$

\begin{tabular}{c|c|c|c}
\hline$h$ & $10^{-1}$ & $10^{-2}$ & $10^{-3}$ \\
\hline RL & $1.4041 \mathrm{e}-01$ & $1.3872 \mathrm{e}-02$ & $1.3778 \mathrm{e}-03$ \\
\hline CF & $1.6750 \mathrm{e}-01$ & $1.7425 \mathrm{e}-02$ & $1.7493 \mathrm{e}-03$ \\
\hline AB & $1.6145 \mathrm{e}-01$ & $1.6430 \mathrm{e}-02$ & $1.6414 \mathrm{e}-03$ \\
\hline
\end{tabular}

(b) $\alpha=0.5$

\begin{tabular}{c|c|c|c}
\hline$h$ & $10^{-1}$ & $10^{-2}$ & $10^{-3}$ \\
\hline RL & $1.4195 \mathrm{e}-01$ & $1.3212 \mathrm{e}-02$ & $1.2740 \mathrm{e}-03$ \\
\hline CF & $1.7875 \mathrm{e}-01$ & $1.8663 \mathrm{e}-02$ & $1.8741 \mathrm{e}-03$ \\
\hline AB & $1.7195 \mathrm{e}-01$ & $1.7387 \mathrm{e}-02$ & $1.7266 \mathrm{e}-03$ \\
\hline \multicolumn{4}{|c}{ (d) $\alpha=1.0$}
\end{tabular}

\begin{tabular}{c|c|c|c}
\hline$h$ & $10^{-1}$ & $10^{-2}$ & $10^{-3}$ \\
\hline RL & $1.4500 \mathrm{e}-01$ & $1.4950 \mathrm{e}-02$ & $1.4995 \mathrm{e}-03$ \\
\hline CF & $1.4500 \mathrm{e}-01$ & $1.4950 \mathrm{e}-02$ & $1.4995 \mathrm{e}-03$ \\
\hline AB & $1.4500 \mathrm{e}-01$ & $1.4950 \mathrm{e}-02$ & $1.4995 \mathrm{e}-03$ \\
\hline
\end{tabular}

Table 2. Experimental order of convergence (EOC) on the basis of absolute relative errors computed at $t=1$ for the example 1

(a) $\alpha=0.25$

\begin{tabular}{c|c|c|c}
\hline$h$ & $1 / 1000$ & $1 / 2000$ & $1 / 4000$ \\
\hline RL & $1.0330 \mathrm{e}+00$ & $1.0286 \mathrm{e}+00$ & $1.0246 \mathrm{e}+00$ \\
\hline CF & $9.9933 \mathrm{e}-01$ & $9.9962 \mathrm{e}-01$ & $9.9982 \mathrm{e}-01$ \\
\hline AB & $1.0042 \mathrm{e}+00$ & $1.0037 \mathrm{e}+00$ & $1.0033 \mathrm{e}+00$ \\
\hline
\end{tabular}
(c) $\alpha=0.75$

\begin{tabular}{c|c|c|c}
\hline$h$ & $1 / 1000$ & $1 / 2000$ & $1 / 4000$ \\
\hline RL & $1.0017 \mathrm{e}+00$ & $1.0011 \mathrm{e}+00$ & $1.0007 \mathrm{e}+00$ \\
\hline CF & $9.9934 \mathrm{e}-01$ & $9.9974 \mathrm{e}-01$ & $9.9985 \mathrm{e}-01$ \\
\hline AB & $1.0005 \mathrm{e}+00$ & $1.0003 \mathrm{e}+00$ & $1.0003 \mathrm{e}+00$ \\
\hline
\end{tabular}

(b) $\alpha=0.5$

\begin{tabular}{c|c|c|c}
\hline$h$ & $1 / 1000$ & $1 / 2000$ & $1 / 4000$ \\
\hline RL & $1.0108 \mathrm{e}+00$ & $1.0078 \mathrm{e}+00$ & $1.0057 \mathrm{e}+00$ \\
\hline CF & $9.9935 \mathrm{e}-01$ & $9.9965 \mathrm{e}-01$ & $9.9982 \mathrm{e}-01$ \\
\hline AB & $1.0025 \mathrm{e}+00$ & $1.0019 \mathrm{e}+00$ & $1.0014 \mathrm{e}+00$ \\
\hline
\end{tabular}

(d) $\alpha=1.0$

\begin{tabular}{c|c|c|c}
\hline$h$ & $1 / 1000$ & $1 / 2000$ & $1 / 4000$ \\
\hline RL & $9.9952 \mathrm{e}-01$ & $9.9977 \mathrm{e}-01$ & $9.9987 \mathrm{e}-01$ \\
\hline CF & $9.9952 \mathrm{e}-01$ & $9.9977 \mathrm{e}-01$ & $9.9987 \mathrm{e}-01$ \\
\hline AB & $9.9952 \mathrm{e}-01$ & $9.9977 \mathrm{e}-01$ & $9.9987 \mathrm{e}-01$ \\
\hline
\end{tabular}

Example 2 Consider $y(t)=\sqrt{t}, t \geq 0$ as a second example for the purpose of comparative analysis. Once again, the exact solution under each integral operator is shown below:

$$
\begin{gathered}
\mathrm{RL}_{J_{0, t}}^{\alpha} y(t)=\frac{\sqrt{\pi} t^{\alpha+1 / 2}}{2 \Gamma(\alpha+3 / 2)} . \\
{ }^{\mathrm{CF}} J_{0, t}^{\alpha} y(t)=\frac{2 \sqrt{t}}{(2-\alpha) M(\alpha)}\left(1-\alpha+\frac{2 \alpha}{3} t\right) . \\
{ }^{\mathrm{AB}} J_{0, t}^{\alpha} y(t)=\frac{1}{B(\alpha)}\left[(1-\alpha) \sqrt{t}+\frac{\alpha \sqrt{\pi} t^{\alpha+1 / 2}}{2 \Gamma(\alpha+3 / 2)}\right] .
\end{gathered}
$$

For this irrational function, one can observe from Table 3 that the absolute relative error computed at the final nodal point $(t=2)$ for an RL fractional integral operator 
is smallest for the fractional-order parameter $\alpha \rightarrow 0$ but otherwise, the AB integral operator yields the smallest error. However, these errors become identical for $\alpha=1$ under each integral operator. Moreover, by decreasing the step length $h$ by one order of magnitude, we get one order decrease in the tenth power of the errors under every integral operator as shown in Table 3. This suggests the first-order convergence of the numerical schemes, which is further confirmed with computation of experimental order of convergence in Table 4 which is always $\approx 1$ but largest in case of the RL fractional integral.

Table 3. Absolute relative errors at final nodal point over $[0,2]$ for the example 2

(a) $\alpha=0.25$

\begin{tabular}{c|c|c|c}
\hline$h$ & $10^{-1}$ & $10^{-2}$ & $10^{-3}$ \\
\hline RL & $2.3917 \mathrm{e}-02$ & $2.1410 \mathrm{e}-03$ & $2.0188 \mathrm{e}-04$ \\
\hline CF & $3.0093 \mathrm{e}-02$ & $2.9202 \mathrm{e}-03$ & $2.8955 \mathrm{e}-04$ \\
\hline AB & $2.4932 \mathrm{e}-02$ & $2.4030 \mathrm{e}-03$ & $2.3672 \mathrm{e}-04$ \\
\hline
\end{tabular}

(c) $\alpha=0.75$

\begin{tabular}{c|c|c|c}
\hline$h$ & $10^{-1}$ & $10^{-2}$ & $10^{-3}$ \\
\hline RL & $3.4061 \mathrm{e}-02$ & $3.2092 \mathrm{e}-03$ & $3.1507 \mathrm{e}-04$ \\
\hline CF & $3.7728 \mathrm{e}-02$ & $3.5876 \mathrm{e}-03$ & $3.5278 \mathrm{e}-04$ \\
\hline AB & $3.2294 \mathrm{e}-02$ & $3.0664 \mathrm{e}-03$ & $3.0192 \mathrm{e}-04$ \\
\hline
\end{tabular}

(b) $\alpha=0.5$

\begin{tabular}{c|c|c|c}
\hline$h$ & $10^{-1}$ & $10^{-2}$ & $10^{-3}$ \\
\hline RL & $2.8007 \mathrm{e}-02$ & $2.5937 \mathrm{e}-03$ & $2.5296 \mathrm{e}-04$ \\
\hline CF & $3.4183 \mathrm{e}-02$ & $3.2777 \mathrm{e}-03$ & $3.2343 \mathrm{e}-04$ \\
\hline AB & $2.6815 \mathrm{e}-02$ & $2.5535 \mathrm{e}-03$ & $2.5166 \mathrm{e}-04$ \\
\hline
\end{tabular}

(d) $\alpha=1.0$

\begin{tabular}{c|c|c|c}
\hline$h$ & $10^{-1}$ & $10^{-2}$ & $10^{-3}$ \\
\hline RL & $4.0830 \mathrm{e}-02$ & $3.8587 \mathrm{e}-03$ & $3.7847 \mathrm{e}-04$ \\
\hline CF & $4.0830 \mathrm{e}-02$ & $3.8587 \mathrm{e}-03$ & $3.7847 \mathrm{e}-04$ \\
\hline AB & $4.0830 \mathrm{e}-02$ & $3.8587 \mathrm{e}-03$ & $3.7847 \mathrm{e}-04$ \\
\hline
\end{tabular}

Table 4. Experimental order of convergence (EOC) on the basis of absolute relative errors computed at $t=2$ for the example 2

(a) $\alpha=0.25$

\begin{tabular}{c|c|c|c}
\hline$h$ & $1 / 1000$ & $1 / 2000$ & $1 / 4000$ \\
\hline RL & $1.0204 \mathrm{e}+00$ & $1.0171 \mathrm{e}+00$ & $1.0143 \mathrm{e}+00$ \\
\hline CF & $1.0023 \mathrm{e}+00$ & $1.0016 \mathrm{e}+00$ & $1.0011 \mathrm{e}+00$ \\
\hline AB & $1.0050 \mathrm{e}+00$ & $1.0042 \mathrm{e}+00$ & $1.0033 \mathrm{e}+00$ \\
\hline \multicolumn{4}{|c}{ (c) $\alpha=0.75$}
\end{tabular}

\begin{tabular}{c|c|c|c}
\hline$h$ & $1 / 1000$ & $1 / 2000$ & $1 / 4000$ \\
\hline RL & $1.0050 \mathrm{e}+00$ & $1.0035 \mathrm{e}+00$ & $1.0025 \mathrm{e}+00$ \\
\hline CF & $1.0047 \mathrm{e}+00$ & $1.0034 \mathrm{e}+00$ & $1.0023 \mathrm{e}+00$ \\
\hline AB & $1.0042 \mathrm{e}+00$ & $1.0030 \mathrm{e}+00$ & $1.0020 \mathrm{e}+00$ \\
\hline
\end{tabular}

(b) $\alpha=0.5$

\begin{tabular}{c|c|c|c}
\hline$h$ & $1 / 1000$ & $1 / 2000$ & $1 / 4000$ \\
\hline RL & $1.0070 \mathrm{e}+00$ & $1.0049 \mathrm{e}+00$ & $1.0036 \mathrm{e}+00$ \\
\hline CF & $1.0037 \mathrm{e}+00$ & $1.0026 \mathrm{e}+00$ & $1.0019 \mathrm{e}+00$ \\
\hline AB & $1.0040 \mathrm{e}+00$ & $1.0028 \mathrm{e}+00$ & $1.0020 \mathrm{e}+00$ \\
\hline \multicolumn{4}{|r}{ (d) $\alpha=1.0$}
\end{tabular}

\begin{tabular}{c|c|c|c}
\hline$h$ & $1 / 1000$ & $1 / 2000$ & $1 / 4000$ \\
\hline RL & $1.0054 \mathrm{e}+00$ & $1.0039 \mathrm{e}+00$ & $1.0028 \mathrm{e}+00$ \\
\hline CF & $1.0054 \mathrm{e}+00$ & $1.0039 \mathrm{e}+00$ & $1.0028 \mathrm{e}+00$ \\
\hline AB & $1.0054 \mathrm{e}+00$ & $1.0039 \mathrm{e}+00$ & $1.0028 \mathrm{e}+00$ \\
\hline
\end{tabular}

Example 3 Consider $y(t)=t \exp (t), t \geq 0$ as a final function to be anlayzed. The exact solution under each integral operator is shown below:

$$
\begin{gathered}
{ }^{\mathrm{RL}} J_{0, t}^{\alpha} y(t)=\frac{1}{\alpha \Gamma(\alpha)}\left[t^{1+\alpha}+(t-\alpha) \exp (t)(\Gamma(1+\alpha)-\Gamma(t, \alpha+1))\right] . \\
{ }^{\mathrm{CF}} J_{0, t}^{\alpha} y(t)=\frac{2}{(2-\alpha) M(\alpha)}[\exp (t)(t-\alpha)+\alpha] .
\end{gathered}
$$


${ }^{\mathrm{AB}} J_{0, t}^{\alpha} y(t)=\frac{1}{B(\alpha)}\left[(1-\alpha) t \exp (t)+\frac{1}{\Gamma(\alpha)}\left[t^{1+\alpha}+(t-\alpha) \exp (t)(\Gamma(1+\alpha)-\Gamma(t, \alpha+1))\right]\right.$.

For this transcendental type of function, one can observe from Table 5 that the absolute relative error computed at the final nodal point $(t=4)$ for the RL fractional integral operator is smallest for varying values of the fractional-order parameter $\alpha$. Varying values of $\alpha$ and $h$ have been used in this example to analyze the behavior of the errors. The first order accuracy is also confirmed with computation of experimental order of convergence in Table 6 which is always $\approx 1$ but largest in case of the RL fractional integral.

Table 5. Absolute relative errors at final nodal point over $[0,4]$ for the example 3 with varying fractional-order parameter $\alpha$ and the step length $h$

(a) $\alpha=0.23$

\begin{tabular}{c|c|c|c}
\hline$h$ & 0.05 & 0.02 & 0.2 \\
\hline RL & $3.9921 \mathrm{e}-02$ & $1.3609 \mathrm{e}-02$ & $1.7157 \mathrm{e}-01$ \\
\hline CF & $5.5534 \mathrm{e}-02$ & $2.2586 \mathrm{e}-02$ & $2.0470 \mathrm{e}-01$ \\
\hline AB & $5.6114 \mathrm{e}-02$ & $2.2270 \mathrm{e}-02$ & $2.1110 \mathrm{e}-01$ \\
\hline
\end{tabular}

(c) $\alpha=0.67$

\begin{tabular}{c|c|c|c}
\hline$h$ & 0.05 & 0.02 & 0.2 \\
\hline RL & $2.7265 \mathrm{e}-02$ & $6.9352 \mathrm{e}-03$ & $1.2927 \mathrm{e}-01$ \\
\hline CF & $4.3796 \mathrm{e}-02$ & $1.7741 \mathrm{e}-02$ & $1.6462 \mathrm{e}-01$ \\
\hline AB & $3.9711 \mathrm{e}-02$ & $1.3556 \mathrm{e}-02$ & $1.6390 \mathrm{e}-01$ \\
\hline
\end{tabular}

(b) $\alpha=0.46$

\begin{tabular}{c|c|c|c}
\hline$h$ & 0.05 & 0.02 & 0.2 \\
\hline RL & $3.0291 \mathrm{e}-02$ & $8.3387 \mathrm{e}-03$ & $1.4263 \mathrm{e}-01$ \\
\hline CF & $4.9755 \mathrm{e}-02$ & $2.0201 \mathrm{e}-02$ & $1.8496 \mathrm{e}-01$ \\
\hline AB & $4.7641 \mathrm{e}-02$ & $1.7687 \mathrm{e}-02$ & $1.8809 \mathrm{e}-01$ \\
\hline \multicolumn{4}{|c}{$\alpha=0.81$}
\end{tabular}

(d) $\alpha=0.81$

\begin{tabular}{c|c|c|c}
\hline$h$ & 0.05 & 0.02 & 0.2 \\
\hline RL & $2.7985 \mathrm{e}-02$ & $8.1226 \mathrm{e}-03$ & $1.2577 \mathrm{e}-01$ \\
\hline CF & $3.9397 \mathrm{e}-02$ & $1.5926 \mathrm{e}-02$ & $1.4960 \mathrm{e}-01$ \\
\hline AB & $3.5421 \mathrm{e}-02$ & $1.1895 \mathrm{e}-02$ & $1.4771 \mathrm{e}-01$ \\
\hline
\end{tabular}

Table 6. Experimental order of convergence (EOC) on the basis of absolute relative errors computed at $t=4$ for the example 3

(a) $\alpha=0.25$

\begin{tabular}{c|c|c|c}
\hline$h$ & $1 / 1000$ & $1 / 2000$ & $1 / 4000$ \\
\hline $\mathrm{RL}$ & $1.0323 \mathrm{e}+00$ & $1.0279 \mathrm{e}+00$ & $1.0239 \mathrm{e}+00$ \\
\hline $\mathrm{CF}$ & $9.9948 \mathrm{e}-01$ & $9.9979 \mathrm{e}-01$ & $9.9990 \mathrm{e}-01$ \\
\hline AB & $1.0044 \mathrm{e}+00$ & $1.0038 \mathrm{e}+00$ & $1.0034 \mathrm{e}+00$ \\
\hline \multicolumn{4}{|c}{$(\mathrm{c}) \alpha=0.75$}
\end{tabular}

(c) $\alpha=0.75$

\begin{tabular}{c|c|c|c}
\hline$h$ & $1 / 1000$ & $1 / 2000$ & $1 / 4000$ \\
\hline RL & $1.0017 \mathrm{e}+00$ & $1.0010 \mathrm{e}+00$ & $1.0007 \mathrm{e}+00$ \\
\hline CF & $9.9954 \mathrm{e}-01$ & $9.9980 \mathrm{e}-01$ & $9.9988 \mathrm{e}-01$ \\
\hline AB & $1.0005 \mathrm{e}+00$ & $1.0004 \mathrm{e}+00$ & $1.0003 \mathrm{e}+00$ \\
\hline
\end{tabular}

(b) $\alpha=0.5$

\begin{tabular}{c|c|c|c}
\hline$h$ & $1 / 1000$ & $1 / 2000$ & $1 / 4000$ \\
\hline RL & $1.0103 \mathrm{e}+00$ & $1.0075 \mathrm{e}+00$ & $1.0054 \mathrm{e}+00$ \\
\hline CF & $9.9957 \mathrm{e}-01$ & $9.9978 \mathrm{e}-01$ & $9.9985 \mathrm{e}-01$ \\
\hline AB & $1.0026 \mathrm{e}+00$ & $1.0020 \mathrm{e}+00$ & $1.0014 \mathrm{e}+00$ \\
\hline
\end{tabular}

(d) $\alpha=1.0$

\begin{tabular}{c|c|c|c}
\hline$h$ & $1 / 1000$ & $1 / 2000$ & $1 / 4000$ \\
\hline RL & $9.9955 \mathrm{e}-01$ & $9.9984 \mathrm{e}-01$ & $9.9983 \mathrm{e}-01$ \\
\hline CF & $9.9955 \mathrm{e}-01$ & $9.9984 \mathrm{e}-01$ & $9.9983 \mathrm{e}-01$ \\
\hline AB & $9.9955 \mathrm{e}-01$ & $9.9984 \mathrm{e}-01$ & $9.9983 \mathrm{e}-01$ \\
\hline
\end{tabular}




\section{Conclusion}

The present study has carried out a comparative analysis for the three most commonly used integrals: Riemann-Liouville, Caputo-Fabrizio, and Atangana-Baleanu integrals. It has been shown that the Riemann-Liouville fractional integral yields the most minor errors while integrating most of the mathematical functions. Furthermore, the computation of the experimental order of convergence (EOC) confirms the firstorder accuracy of the schemes under consideration for all three types of integrals. The final observation suggests that the Caputo-Fabrizio integral operator is a linear combination of $y(t)$ and an ordinary integral. In contrast, the Atangan-Baleanu is also a linear combination of $y(t)$ and the Riemann-Liouville fractional integral itself. It is worth mentioning that the Atangana-Baleanu, despite being represented in linear combination with the RL integral, has proved to be an essential operator to solve several dynamical systems that could not be handled with an RL operator alone. It is also observed from the present published research that all these three integral operators have importance in several scientific fields, including dynamical systems, chaos theory, epidemiology, feedback control systems, and applied mathematics.

\section{References}

[1] Jaradat, I., Al-Dolat, M., Al-Zoubi, K., \& Alquran, M. (2018). Theory and applications of a more general form for fractional power series expansion. Chaos, Solitons \& Fractals, 108, 107-110.

[2] Alquran, M., \& Jaradat, I. (2018). A novel scheme for solving Caputo time-fractional nonlinear equations: theory and application. Nonlinear Dynamics, 91(4), 2389-2395.

[3] Alquran, M., Yousef, F., Alquran, F., Sulaiman, T.A., \& Yusuf, A. (2021). Dual-wave solutions for the quadratic-cubic conformable-Caputo time-fractional Klein-Fock-Gordon equation. Mathematics and Computers in Simulation, 185, 62-76.

[4] Alquran, M., Al-Khaled, K., Sivasundaram, S., \& Jaradat, H.M. (2017). Mathematical and numerical study of existence of bifurcations of the generalized fractional Burgers-Huxley equation. Nonlinear Studies, 24(1), 235-244.

[5] Yusuf, A., Qureshi, S., Inc, M., Aliyu, A.I., Baleanu, D., \& Shaikh, A.A. (2018). Two-strain epidemic model involving fractional derivative with Mittag-Leffler kernel. Chaos: An Interdisciplinary Journal of Nonlinear Science, 28(12), 123121.

[6] Zhang, L., Rahman, M.U., Ahmad, S., Riaz, M.B., \& Jarad, F. (2022). Dynamics of fractional order delay model of coronavirus disease. AIMS Mathematics, 7(3), 4211-4232.

[7] Ur Rahman, M., Arfan, M., Shah, K., \& Gómez-Aguilar, J.F. (2020). Investigating a nonlinear dynamical model of COVID-19 disease under fuzzy Caputo, random and ABC fractional order derivative. Chaos, Solitons \& Fractals, 140, 110232.

[8] Yusuf, A., Qureshi, S., \& Shah, S.F. (2020). Mathematical analysis for an autonomous financial dynamical system via classical and modern fractional operators. Chaos, Solitons \& Fractals, 132,109552

[9] Rahman, M.U., Arfan, M., Deebani, W., Kumam, P., \& Shah, Z. (2021). Analysis of timefractional Kawahara equation under Mittag-Leffler power law. Fractals, 2240021.

[10] Ur Rahman, M., Arfan, M., Shah, K., \& Gómez-Aguilar, J.F. (2020). Investigating a nonlinear dynamical model of COVID-19 disease under fuzzy Caputo, random and ABC fractional order derivative. Chaos, Solitons \& Fractals, 140, 110232. 
[11] Ur Rahman, M., Arfan, M., Shah, Z., \& Alzahrani, E. (2021). Evolution of fractional mathematical model for drinking under Atangana-Baleanu Caputo derivatives. Physica Scripta, 96(11), 115203

[12] Qureshi, S. (2021). Fox H-functions as exact solutions for Caputo type mass spring damper system under Sumudu transform. Journal of Applied Mathematics and Computational Mechanics, 20(1), 83-89.

[13] Abro, K.A., Qureshi, S., \& Atangana, A. (2020). Mathematical and numerical optimality of nonsingular fractional approaches on free and forced linear oscillator. Nonlinear Engineering, 9(1), 449-456.

[14] Qureshi, S. (2021). Fox H-functions as exact solutions for Caputo type mass spring damper system under Sumudu transform. Journal of Applied Mathematics and Computational Mechanics, 20(1), 83-89.

[15] Qureshi, S., Chang, M.M., \& Shaikh, A.A. (2021). Analysis of series RL and RC circuits with time-invariant source using truncated M, Atangana beta and conformable derivatives. Journal of Ocean Engineering and Science, 6(3), 217-227.

[16] Kulish, V.V., \& Lage, J.L. (2002). Application of fractional calculus to fluid mechanics. Journal of Fluids Engineering, 124(3), 803-806.

[17] Monje, C.A., Chen, Y., Vinagre, B.M., Xue, D., \& Feliu-Batlle, V. (2010). Fractional-order Systems and Controls: Fundamentals and Applications. Springer Science \& Business Media.

[18] Lopes, A.M., \& Machado, J.T. (2014). Fractional order models of leaves. Journal of Vibration and Control, 20(7), 998-1008.

[19] Lopes, A.M., Machado, J.T., \& Ramalho, E. (2017). On the fractional-order modeling of wine. European Food Research and Technology, 243(6), 921-929.

[20] Biswas, K., Bohannan, G., Caponetto, R., Lopes, A.M., \& Machado, J.A.T. (2017). Fractionalorder models of vegetable tissues. Fractional-Order Devices, 73-92. DOI: 10.1007/978 - 3 $319-54460-1_{4}$.

[21] Caputo, M., \& Fabrizio, M. (2015). A new definition of fractional derivative without singular kernel. Progr. Fract. Differ. Appl., 1(2), 1-13.

[22] Atangana, A., \& Baleanu, D. (2016). New fractional derivatives with nonlocal and non-singular kernel: theory and application to heat transfer model. Thermal Science, 20, 2, 763-769. DOI: $10.2298 /$ TSCI160111018A.

[23] Li, C., \& Zeng, F. (2019). Numerical Methods for Fractional Calculus. Chapman and Hall/CRC.

[24] Diethelm, K., Ford, N.J., \& Freed, A.D. (2004). Detailed error analysis for a fractional Adams method. Numerical Algorithms, 36(1), 31-52.

[25] Burden, R.L., \& Faires, J.D. (1997). Numerical Analysis. Cole, Belmont. 